\title{
Large Time Behavior of the Vorticity of Two-Dimensional Viscous Flow and its Application to Vortex Formation
}

\author{
Yoshikazu Giga ${ }^{1}$ and Tsutomu Kambe ${ }^{2 \star}$ \\ ${ }^{1}$ Department of Mathematics, Hokkaido University, Sapporo 060, Japan \\ ${ }^{2}$ Department of Physics, University of Tokyo, Tokyo 113, Japan
}

\begin{abstract}
We consider the Cauchy problem for the two-dimensional vorticity equation. We show that the solution $\omega$ behaves like a constant multiple of the Gauss kernel having the same total vorticity as time tends to infinity. No particular structure of initial data $\omega_{0}=\omega(x, 0)$ is assumed except the restriction that the Reynolds number $R=\int\left|\omega_{0}\right| d x / v$ is small, where $v$ is the kinematic viscosity. Applying a time-dependent scale transformation, we show a stability of Burgers' vortex, which physically implies formation of a concentrated vortex.
\end{abstract}

\section{Introduction}

This paper studies the large time asymptotic behavior of the vorticity distribution of two-dimensional viscous incompressible flow. We consider the two-dimensional vorticity equation

$$
\frac{\partial \omega}{\partial t}-v \Delta \omega+(v \cdot \nabla) \omega=0, \quad v=K * \omega,
$$

which is known to be equivalent to the Navier-Stokes equations. Here $\omega=\omega(x, t)$ and $v=\left(v^{1}(x, t), v^{2}(x, t)\right)$ represent the scalar vorticity distribution and the velocity field, respectively; $v>0$ is the kinematic viscosity and $v \cdot \nabla=\sum_{j=1}^{2} v^{j} \partial / \partial x_{j}$. The second equation involving the convolution $*$ is called the Biot-Savart law. Its explicit form is

$$
v(x, t)=\int_{\mathbb{R}^{2}} K(x-y) \omega(y, t) d y,
$$

where $K$ is the vector function

$$
K\left(x_{1}, x_{2}\right)=\left(-x_{2}, x_{1}\right) / 2 \pi|x|^{2}, \quad x=\left(x_{1}, x_{2}\right) .
$$

* Partly supported by Grant-in-Aid for Scientific Research No. B60460042, the Japan Ministry of Education, Science and Culture 
There is a special solution to (1) called Oseen's vortex [17],

$$
\omega_{* \kappa}(x, t)=\frac{\kappa}{4 \pi v t} \exp \left(-\frac{|x|^{2}}{4 v t}\right) \quad(\kappa: \text { real number }),
$$

where $\kappa$ is the strength of the vortex. Since $\omega_{* \kappa}$ is a Gauss kernel, $\omega_{* \kappa}$ is regarded as a solution of (1) with the initial data $\omega_{* \kappa}(x, 0)=\kappa \delta(x)$, where $\delta(x)$ is Dirac's delta function.

The main goal of this paper is to show that even if we start with a general initial vorticity distribution $\omega_{0}$, the solution $\omega$ of (1) behaves like the above special solution $\omega_{* \kappa}$ as $t \rightarrow \infty$ with the total vorticity $\kappa=\int \omega_{0} d x$, provided that the Reynolds number $R=\int\left|\omega_{0}\right| d x / v$ is sufficiently small. In fact, we prove

$$
\left\|\omega-\omega_{* \kappa}\right\|_{p}(t) \leqq C(v t)^{-1+1 / p-\delta} \quad t>0, \quad 1 \leqq p \leqq \infty,
$$

for $0<\delta<1 / 2$, provided that $R$ is sufficiently small and that $\int\left|\omega_{0}\right|\left(|x|^{2}+1\right) d x$ is finite where $C$ is a constant independent of $t$ and $v ;\|\cdot\|_{p}$ denotes the $L^{p}$-norm in space variables. Since $\left\|\omega_{* \kappa}\right\|_{p}(t)=C_{p} \kappa(v t)^{-1+1 / p},\left(C_{p}\right.$ : constant depending only on $p$ ), our estimate (3) gives an asymptotic expression of $\omega$ as $t \rightarrow \infty$. No particular structure of initial vorticity $\omega_{0}$ is assumed.

As an application of (3) we show a stability of Burgers' vortex [5], which physically implies formation of a concentrated vortex. We consider a threedimensional viscous incompressible flow expressed as a superposition of two flows - an axisymmetric irrotational flow and a two-dimensional flow the vorticity of which directs to the symmetry axis. The axisymmetric flow is assumed to have an inward convection and axially stretching flow which is an incompressible flow with constant rate of strain. We show that the vorticity field tends to its equilibrium state called Burgers' vortex as the time tends to infinity, provided that the Reynolds number $R$ of the rotational part is sufficiently small. In fact the three-dimensional vorticity equations can be transformed to (1) by a time-dependent scale transformation due to Kambe $[11,13]$ and Lundgren [16]. Such an asymptotic behavior is shown by Kambe [12] assuming that initial vorticity is axially symmetric, but for arbitrary $R$ since the governing equation (1) is reduced to the heat equation. Our results extend this because no particular structure of initial vorticity is assumed. Although we are forced to assume that $R$ is small, we do not restrict the speed of the axisymmetric irrotational flow.

To prove (3) we study the integral form of (1):

$$
\omega(t)=e^{v t \Delta} \omega_{0}+B(\omega, \omega), \quad B(\omega, \omega)=-\int_{0}^{t} e^{v(t-s) \Delta}(v \cdot \nabla) \omega(s) d s,
$$

where $U=e^{v t} \Delta \omega_{0}$ solves the heat equation $\partial_{t} U-v \Delta U=0$ with $U(x, 0)=\omega_{0}$ and $\omega(t)=\omega(\cdot, t)$. Unfortunately, the term $B(\omega, \omega)$ cannot be regarded as a minor term as $t \rightarrow \infty$, unless we use the special structure of $B$. Since $\omega_{* \kappa}$ defined in (2) is radially symmetric, as is easily seen $\omega_{* \kappa}$ solves (1) with $(v \cdot \nabla) \omega=0$. This implies $B\left(\omega_{* \kappa}, \omega_{* \kappa}\right)=0$. Applying this property to (4) we rewrite the equation for the difference $w=\omega-\omega_{* \kappa}$ to obtain

$$
w=W+B(w, w)+B\left(w, \omega_{* \kappa}\right)+B\left(\omega_{* \kappa}, w\right), \quad W=e^{v t} \Delta_{0}-\omega_{* \kappa} .
$$


We estimate the right-hand side of (5). Based on the decay estimate

$$
\|\omega\|_{p}(t) \leqq C R t^{-1+1 / p}
$$

obtained by Giga, Miyakawa and Osada [8], one can regard B's in (5) as perturbation terms provided that $R$ is sufficiently small. Thus the estimate for $w$ in (5) is reduced to the estimate for $W$ which is easy to derive. As is seen above, our result (3) heavily depends on the particular structure of the nonlinear term in (1) and the estimate (6).

It turns out that the estimate (3) is still valid even if we allow to choose a finite Radon measure as initial vorticity rather than integrable functions. Since $\omega(x, t)$ is regularized instantaneously, this is not a substantial improvement of the results. However, all estimates are parallel and there appears to be no extra difficulty. So we rather start with a finite Radon measure because the initial value of $\omega_{* \kappa}$ is $\kappa \delta(x)$, which is a typical example of a finite Radon measure but not an integrable function. We note that vortex sheets of finite length is another example of finite Radon measures.

There are many works on the large time behavior of solutions of the Navier-Stokes equations on $\mathbb{R}^{n}$ (cf. $\left.[2,10,14,18,20-22]\right)$. However, when $n=2$ it is usually assumed that initial velocity $v_{0}=K * \omega_{0}$ is in $L^{2}\left(\mathbb{R}^{2}\right)$, in other words the initial total energy is finite. Our assumption does not imply $v_{0} \in L^{2}\left(\mathbb{R}^{2}\right)$ even if we assume $\omega_{0} \in L^{1}\left(\mathbb{R}^{2}\right)$. So our situation is not included in those treated in the literature. In our setting even the existence of the solution of (1) is recently proved in [8] with a decay estimate (6). The decay results in the literature is mostly not for the vorticity but the velocity, especially its $L^{2}$-norm. For more detailed comparison with the literature, see Remark 4.4.

We study in Sect. 2 the asymptotic behavior of the solutions of the heat equation so that we estimate the decay of $W$ in (5). In Sect. 3 we recall the estimate (6) and prepare estimates for $B$. In Sect. 4 we state our main results including (3), which are proved in Sect. 5. The final section is devoted to an application of our results in Sect. 3, which is mentioned in the third paragraph of the Introduction.

\section{The Heat Equation}

The goal of this section is to prepare various estimates for the solution of the heat equation as time $t$ tends to infinity. We are especially concerned with estimates for the second term in the asymptotic expansion of the solution as $t \rightarrow \infty$.

Let $G$ be the Gauss kernel on $\mathbb{R}^{n}$ defined by

$$
G(x, t)=\frac{1}{(4 \pi t)^{n / 2}} \exp \left(-\frac{|x|^{2}}{4 t}\right), \quad x \in \mathbb{R}^{n}, \quad t>0 .
$$

As is well known the function

$$
U(x, t)=\int_{\mathbb{R}^{n}} G(x-y, t) a(y) d y(=G * a)
$$

solves the heat equation

$$
\partial_{t} U-\Delta U=0 \text { for } t>0
$$


with

$$
U(x, 0)=\lim _{t \rightarrow+0} U(x, t)=a(x) .
$$

The meaning of the convergence of $U(x, t)$ as $t \rightarrow+0$ depends on a class of functions $a$. We shall write $U$ by $e^{t \Delta} a$. The semigroup $e^{t \Delta}$ is used only for the convenience of notation; we shall not use the abstract theory of semigroups in this paper.

We collect various estimates for $G\left(=e^{t \Delta} \delta\right)$ and $e^{t \Delta} a$. A direct calculation shows

$$
\begin{aligned}
\|G\|_{r} & =\left(\int_{\mathbb{R}^{n}}|G|^{r} d x\right)^{1 / r}=C_{r} t^{-\sigma(r)}, \quad \sigma(r)=\frac{n}{2}\left(1-\frac{1}{r}\right), \\
\left\|\partial_{j} G\right\|_{r} & =C_{r}^{\prime} t^{-\sigma(r)-1 / 2}, \quad \partial_{j}=\partial / \partial x_{j},
\end{aligned}
$$

where $C_{r}$ and $C_{r}^{\prime}$ are constants depending only on $n$ and $r$. When $r=\infty$, (2.1a) and (2.1b) still hold if we regard $\|f\|_{\infty}$ as the supremum of $|f|$ on $\mathbb{R}^{n}$ and $\sigma(\infty)=n / 2$. Applying the Young inequality.

$$
\|f * g\|_{p} \leqq\|f\|_{r}\|g\|_{q}
$$

for $1 / p=1 / r+1 / q-1,1 \leqq p, q, r \leqq \infty$ with $f=G, g=a$, we see $(2.1 \mathrm{a}, \mathrm{b})$ yields

$$
\begin{aligned}
& \left\|e^{t \Delta} a\right\|_{p} \leqq C t^{-(n / q-n / p) / 2}\|a\|_{q}, \quad t>0, \quad 1 \leqq q \leqq p \leqq \infty, \\
& \left\|\partial_{j} e^{t \Delta} a\right\|_{p} \leqq C t^{-(n / q-n / p) / 2-1 / 2}\|a\|_{q}, \quad t>0, \quad 1 \leqq q \leqq p \leqq \infty,
\end{aligned}
$$

where $C=\max \left(\sup _{r} C_{r}, \sup _{r} C_{r}^{\prime}\right)$ depends only on $n$. This gives a decay estimate for $e^{t \Delta} a$ as $t \rightarrow \infty$, provided $a$ is in $L^{q}\left(\mathbb{R}^{n}\right)$. In particular, (2.3a) yields

$$
\left\|e^{t \Delta} a\right\|_{p} \leqq C t^{-\sigma(p)}\|a\|_{1} \quad t>0, \quad 1 \leqq o \leqq \infty .
$$

This estimate extends to a finite Radon measure $a$ on $\mathbb{R}^{n}$. A finite Radon measure $\mu$ is a Schwartz distribution which is a bounded linear functional on the Banach space $B C\left(\mathbb{R}^{n}\right)$ of bounded continuous functions on $\mathbb{R}^{n}$. The space of finite Radon measures on $\mathbb{R}^{n}$ denotes $M\left(\mathbb{R}^{n}\right)$. The norm of $\mu$ in $M\left(\mathbb{R}^{n}\right)$ is called the total variation of $\mu$ denoted by $\|\mu\|_{1}$. Its explicit definition is

$$
\|\mu\|_{1}=\sup _{\substack{\phi \in B C\left(\mathbb{R}^{n}\right) \\\|\phi\|_{\infty} \leqq 1}}\left|\int_{\mathbb{R}^{n}} \phi(x) \mu(d x)\right|,
$$

where $\int \phi(x) \mu(d x)$ denotes the canonical duality pairing. The estimate (2.4) holds for $a \in M\left(\mathbb{R}^{n}\right)$ if we regard $\|a\|_{1}$ as the total variation of $a$. In fact, (2.2) is valid for a Radon measure $g$ by setting $q=1$ and $p=r$.

We next approximate $e^{t \Delta} a$ by $\alpha G$ with $\alpha=\int a(x) d x$ for large $t$. Formally,

$$
\begin{aligned}
\left(e^{t \Delta} a\right)(x) & =\int_{\mathbb{R}^{n}} G(x-y, t) a(y) d y=G(x) \int_{\mathbb{R}^{n}} \exp \left(\frac{2 x \cdot y-|y|^{2}}{4 t}\right) a(y) d y \\
& =\alpha G+0\left(t^{-n / 2-1}\right) \quad \text { as } t \rightarrow \infty \text { (pointwise), }
\end{aligned}
$$

since $\exp \left(\left(2 x \cdot y-y^{2}\right) / 4 t\right)=1+\left(2 x \cdot y-y^{2}\right) / 4 t+\cdots$. We give a rigorous meaning for this approximation. Since we are interested in uniform estimates in $x$, we lose $t^{-1 / 2}$ in estimating the error term. 
Lemma 2.1. Assume that $1 \leqq p \leqq \infty$ and that a is a finite Radon measure on $\mathbb{R}^{n}$. Let $\alpha=\int_{\mathbb{R}^{n}} a(d x)$. Then

$$
\left\|e^{t \Delta} a-\alpha G\right\|_{p} \leqq C t^{-\sigma(p)}\left(t^{-1 / 2}\||x| a\|_{1}+t^{-1}\left\||x|^{2} a\right\|_{1}\right), \quad t>0,
$$

where $\sigma(p)=n(1-1 / p) / 2$ and $C$ depends only on $n$. In particular,

$$
\left\|e^{t \Delta} a-\alpha G\right\|_{p} \leqq C t^{-\sigma(p)-1 / 2}\left\|\left(|x|^{2}+1\right) a\right\|_{1} \text { for } t>0 .
$$

Proof. The idea of the proof is standard. Estimates similar to those used in the proof often appear in constructing the fundamental solutions of parabolic equations (see eg. $[6,15]$ ). However, since the proof is short we give it here for completeness. We write the proof pretending that the measure has the density, i.e., $a \in L^{1}\left(\mathbb{R}^{n}\right)$ only to use a standard calculus notation. The proof is the same for general $a \in M\left(\mathbb{R}^{n}\right)$ if we modify notation by replacing $a(y) d y,|a(y)| d y$ by $a(d y),|a|(d y)$, respectively, since all functions appearing below are continuous in each variable for $t>0$.

Since $\int G d x=1$ we see

with

$$
e^{t \Delta} a-\alpha G=\int h(x, y, t) a(y) d y
$$

$$
h(x, y, t)=G(x-y, t)-G(x, t),
$$

where the integration is over $\mathbb{R}^{n}$. By the mean value theorem we have

$$
\begin{aligned}
(4 \pi t)^{n / 2} h(x, y, t) & =\left(\exp \left(\frac{2 x \cdot y-|y|^{2}}{4 t}\right)-1\right) \exp \left(-\frac{|x|^{2}}{4 t}\right) \\
& =\frac{2 x \cdot y-|y|^{2}}{4 t} \cdot \exp \left(-\frac{|x|^{2}-\zeta}{4 t}\right),
\end{aligned}
$$

where $\zeta$ is a number between zero and $2 x \cdot y-|y|^{2}$. If $2 x \cdot y-|y|^{2} \geqq 0$, then $\zeta \leqq 2 x \cdot y-|y|^{2}$, which yields

$$
|x|^{2}-\zeta \geqq|x-y|^{2}
$$

Applying this to (2.7) gives

$$
4(4 \pi t)^{n / 2}|h| \leqq t^{-1}\left(2(x-y) \cdot y+|y|^{2}\right) \exp \left(-\frac{|x-y|^{2}}{4 t}\right) .
$$

Since $A_{\beta}=\sup _{z>0} z \exp \left(-\beta z^{2} / 4\right)<\infty$ for $\beta>0$, we see

$$
\left(|x-y| / t^{1 / 2}\right) \exp \left(-\frac{\beta|x-y|^{2}}{4 t}\right) \leqq A_{\beta} .
$$

We take $\beta$ such that $0<\beta<1$, and fix $\beta$ to obtain

$$
\begin{aligned}
|h(x, y, t)| & \leqq k(y, t) G(x-y, \gamma t), \\
k(y, t) & =\left(A_{\beta}|y| / 2 t^{1 / 2}+|y|^{2} / 4 t\right) \gamma^{n / 2}, \quad \gamma=1 /(1-\beta),
\end{aligned}
$$

provided $2 x \cdot y-|y|^{2} \geqq 0$. If $2 x \cdot y-|y|^{2}<0$, we see $\zeta<0$. This time (2.7) yields

$$
4(4 \pi t)^{n / 2}|h| \leqq\left(|y|^{2} / t+2|x||y| / t\right) \exp \left(-\frac{|x|^{2}}{4 t}\right) .
$$


As before we obtain

$$
|h(x, y, t)| \leqslant k(y, t) G(x, \gamma t)
$$

provided $2 x \cdot y-|y|^{2}<0$.

The estimates (2.8) and (2.9) yield

$$
F(x)=\left|\int h a d y\right| \leqq \max \left(e^{\gamma t \Delta}(k|a|), \quad G(x, \gamma t) \cdot \int k|a| d y\right) .
$$

Applying (2.4) and (2.1a) gives

$$
\|F(x)\|_{p} \leqq C t^{-\sigma(p)}\|k|a|\|_{1}
$$

with $C$ depending only on $n$. This is the same as $(2.5)$, since $k \leqq C\left(|y| / t^{1 / 2}+|y|^{2} / t\right)$. The estimate (2.6) for $t \geqslant 1$ follows from (2.5). Since $|\alpha| \leqq\|a\|_{1}$, the estimate (2.6) for $t \leqq 1$ follows from (2.1a) and (2.3a) with $t \leqq 1$. We thus obtain (2.6).

\section{The Two-Dimensional Vorticity Equation}

The first part of this section reviews the existence of solutions to the twodimensional vorticity equation with measures as initial vorticity. We also recall decay estimates for the vorticity as time tends to infinity.

The second part is devoted to the study of properties of the nonlinear term in the vorticity equation which is useful in the sequel.

Existence results mentioned above are not classical because the initial velocity may not be locally square summable even if initial vorticity is in $L^{1}\left(\mathbb{R}^{2}\right)$. The first attack is done by Benfatto, Esposito and Pulvirenti [3], where they prove the global existence for small initial vorticity consisting of a linear combination of $\delta$-functions supported at a point of the plane. Recently, Miyakawa, Osada and the first author [8] improve their results. Without the smallness assumption they [8] constructed a global solution even if initial vorticity is a finite Radon measure, and also give decay estimates for the vorticity as time tends to infinity.

We consider the two-dimensional vorticity equation:

$$
\begin{gathered}
\omega_{t}-\Delta \omega+(v \cdot \nabla) \omega=0, \\
v=K * \omega,
\end{gathered}
$$

where $K$ is the vector function

$$
K\left(x_{1}, x_{2}\right)=\left(-x_{2}, x_{1}\right) / 2 \pi|x|^{2}, \quad x=\left(x_{1}, x_{2}\right) ;
$$

we shall always assume the space dimension $n=2$. Here the vorticity $\omega=\omega(x, t)$ is scalar since $n=2$. The convolution operator $K *$ improves differentiability of order one. Another expression of this regularizing property of $K *$ is

$$
\|K * f\|_{p} \leqq C\|f\|_{q} \quad 1 / q=1 / p+1 / 2, \quad 1<q<2,
$$

obtained by the Hardy-Littlewood-Sobolev inequality (see e.g. [19, p. 28]), where $\|f\|_{q}$ denotes the norm of $f$ in $L^{q}\left(\mathbb{R}^{2}\right)$ and $C$ depends only on $q$. We consider (3.1) and (3.2) with initial condition

$$
\omega(x, 0)=\lim _{t \rightarrow+0} \omega(x, t)=\omega_{0}(x) .
$$


If $\omega_{0}$ is a finite Radon measure on $\mathbb{R}^{2}$, i.e., $\omega_{0} \in M\left(\mathbb{R}^{2}\right)$, the convergence should be understood under the weak topology of measures, that is,

$$
\lim _{t \rightarrow+0} \int_{\mathbb{R}^{2}} \phi(x) \omega(x, t) d x=\int_{\mathbb{R}^{2}} \phi(x) \omega_{0}(d x)
$$

for every bounded continuous functions $\phi$ on $\mathbb{R}^{2}$. We write $\omega(x, t) d x$ rather than $\omega(d x, t)$ because the solution $\omega$ we handle is smooth for $t>0$.

Proposition 3.1. Suppose that $\omega_{0} \in M\left(\mathbb{R}^{2}\right)$, i.e. $\omega_{0}$ is a Radon measure on $\mathbb{R}^{2}$ such that the total variation $\left\|\omega_{0}\right\|_{1}$ is finite. There is a global solution of (3.1), (3.2) and (3.4) such that

$$
\begin{gathered}
\|\omega\|_{q}(t) \leqq C t^{-1+1 / q}\left\|\omega_{0}\right\|_{1} \quad t>0, \quad 1 \leqq q \leqq \infty \\
\|v\|_{p}(t) \leqq C^{\prime} t^{-1 / 2+1 / p}\left\|\omega_{0}\right\|_{1} \quad t>0, \quad 2<p \leqq \infty \\
\sup _{t \leqq \tau \leqq T}\left\|\nabla^{k} \partial_{t}^{h} \omega\right\|_{\infty}(\tau)<\infty, \quad k, h=0,1,2, \ldots \quad t>0 \\
\int_{\mathbb{R}^{2}} \omega(x, t) d x=\int_{\mathbb{R}^{2}} \omega_{0}(d x), \quad t>0
\end{gathered}
$$

with $C=C(m), C^{\prime}=C^{\prime}(m, p)$ for $\left\|\omega_{0}\right\|_{1} \leqq m$. The estimate (3.7) in particular implies that $\omega$ is smooth for $t>0$.

Proof. This is essentially a combination of Theorems 4.2 and 4.3 in [8] with initial velocity $u_{0}=K * \omega_{0}$. In fact, Theorem 4.2 in [8] asserts that there is a smooth solution $u(x, t)$ for $t>0$ to the Navier-Stokes system:

$$
u_{t}-\Delta u+(u \cdot \nabla) u+\nabla p=0, \quad \nabla \cdot u=0
$$

with initial velocity $u_{0}$ and initial vorticity $\omega_{0}$. Taking $\nabla \times$ of the first equation shows that $\omega=\nabla \times u$ with $v=u$ solves (3.1) for $t>0$. Since Theorem 4.2 (4.1) in [8] implies $\|u\|_{r}(t)<\infty$ for $t>0, r>2$, applying Lemma 2.2(ii) in [8], we see $\omega=\nabla \times u$ solves (3.2) with $v=u$. We thus conclude that $\omega=\nabla \times u, v=u$ solves (3.1)-(3.2) for $t>0$, where $u$ is in Theorem 4.2 [8].

The initial condition (3.4) is contained in Theorem 4.2(ii) of [8]. The estimate (3.5) is the same as (4.7) of Theorem 4.3 in [8]. This together with (3.3) yields (3.6). The estimate (3.7) immediately follows from Theorem 4.2(iv) in [8]. Theorem 4.3 in [8] also gives a representation for $\omega$,

$$
\omega(x, t)=\int \Gamma(x, t ; y, 0) \omega_{0}(d y)
$$

with $\Gamma(x, t, y, s)>0$ and $\int \Gamma(x, t, y, s) d x=1$, where the integration is over $\mathbb{R}^{2}$. This yields the conservation of the vorticity (3.8). (We note that the representation for $\omega$ together with estimates for $\Gamma$ yields (3.5).)

Remark 3.2. If the point vortex part of initial measure $\omega_{0}$ is small we have the uniqueness of solution in a certain class of functions [8]. In particular, if $\left\|\omega_{0}\right\|_{1}$ is small or $\omega_{0}$ contains no point vortices, one can claim the uniqueness with additional conditions (cf. Theorem 4.5 in [8]). For general initial data in $M\left(\mathbb{R}^{2}\right)$ we do not know what conditions guarantee the uniqueness of solutions. However, since our $\omega$ in Proposition 3.1 has as physically reasonable properties as (3.8), by 
a solution of the vorticity equation, we shall always mean $\omega(x, t)$ in Proposition 3.1.

It is convenient to write Eqs. (3.1), (3.2) and (3.4) in its integral form. Formally the system (3.1), (3.2), (3.4) is equivalent to

$$
\begin{aligned}
\omega(t) & =e^{t \Delta} \omega_{0}+B(\omega, \omega), \\
B(\omega, \omega) & =\int_{0}^{t} b_{t}(\omega, \omega)(s) d s, \\
b_{t}\left(\omega_{1}, \omega_{2}\right)(s) & =-\nabla \cdot e^{(t-s) \Delta}\left(v_{1} \omega_{2}\right)(s), \quad v_{1}=K * \omega_{1} \\
& =-\sum_{j=1}^{2} \partial_{j} e^{(t-s)} v_{1}^{j}(s) \omega_{2}(s),
\end{aligned}
$$

since $\nabla \cdot v=0$ implies $\nabla \cdot(v \omega)=(v \cdot \nabla) \omega$, and since $\nabla$ commutes with $e^{t \Delta}$. Here $e^{t \Delta}$ is the solution operator of the heat equation defined in Sect. 2, i.e., $e^{t \Delta} f=G * f$, and $\omega(t)=\omega(\cdot, t)$.

Proposition 3.3. Suppose that $\omega(x, t)$ is a solution of the vorticity equation, where $\omega(x, 0)=\omega_{0} \in M\left(\mathbb{R}^{2}\right)$. Then $\left\|b_{t}(\omega, \omega)\right\|_{1}(s)$ is integrable on $(0, t)$, where $b_{t}$ is defined in (3.10). Moreover $\omega(t)=\omega(\cdot, t)$ solves the integral equation (3.9).

Proof. Applying (2.1b) yields

$$
\left\|b_{t}(\omega, \omega)(s)\right\|_{1} \leqq C(t-s)^{-1 / 2}\|v \omega\|_{1} .
$$

The estimates (3.5) and (3.6) now show that $\left\|b_{t}(\omega, \omega)(s)\right\|_{1}$ is integrable on $(0, t)$.

It remains to prove that $\omega$ solves (3.9). By (3.7) a classical uniqueness theorem of solutions of the heat equation [6] shows that

$$
\omega(t)=e^{(t-\varepsilon) 4} \omega(\varepsilon)+\int_{\varepsilon}^{t} b_{t}(\omega, \omega)(s) d s
$$

for $\varepsilon>0$. We shall show that for $t>0$, $e^{(t-\varepsilon) \Delta} \omega(\varepsilon) \rightarrow e^{t \Delta} \omega_{0}$ weakly in $M\left(\mathbb{R}^{2}\right)$ as $\varepsilon \rightarrow 0$, i.e.

$$
\lim _{\varepsilon \rightarrow 0}\left(\phi, e^{(t-\varepsilon) \Delta} \omega(\varepsilon)\right)=\left(\phi, e^{t \Delta} \omega_{0}\right)
$$

for every bounded continuous function $\phi$, where $(\phi, \psi)=\int \phi \psi d x$. We have

$$
\begin{aligned}
\left(e^{(t-\varepsilon) \Delta} \omega(\varepsilon)-e^{t \Delta} \omega_{0}, \phi\right) & =\left(\left(e^{(t-\varepsilon) \Delta}-e^{t \Delta}\right) \omega(\varepsilon), \phi\right)+\left(e^{t \Delta}\left(\omega(\varepsilon)-\omega_{0}\right), \phi\right) \\
& =\left(e^{(t-\varepsilon) \Delta} \omega(\varepsilon),\left(I-e^{\varepsilon \Delta}\right) \phi\right)+\left(\omega(\varepsilon)-\omega_{0}, e^{t \Delta} \phi\right)=I_{1}+I_{2} .
\end{aligned}
$$

Using (2.4) and (3.5), the first term is estimated as

$$
\left|I_{1}\right| \leqq C\left\|\omega_{0}\right\|_{1}\left\|\left(I-e^{\varepsilon \Delta}\right) \phi\right\|_{\infty} .
$$

Since $e^{\varepsilon} \phi \rightarrow \phi$ uniformly as $\varepsilon \rightarrow 0, I_{1} \rightarrow 0$ as $\varepsilon \rightarrow 0$. Since $\omega(\varepsilon) \rightarrow \omega_{0}$ weakly in $M\left(\mathbb{R}^{2}\right)$ as $\varepsilon \rightarrow 0$, we see $I_{2} \rightarrow 0$ as $\varepsilon \rightarrow 0$. We thus obtain (3.12). Since $\left\|b_{t}(\omega, \omega)(s)\right\|_{1}$ is integrable on $(0, t),(3.12)$ now yields (3.9) by letting $\varepsilon \rightarrow 0$ in (3.11).

The remaining part of this section is devoted to the study of $B$ defined in (3.10).

Lemma 3.4. Let $b_{t}$ and $B$ be the bilinear forms defined in (3.10). Then $(K * f \cdot \nabla) g=0$, 
so $b_{t}(f, g)=0$ and $B(f, g)=0$, provided that $f$ and $g$ are radially symmetric, where $K=\left(-x_{2}, x_{1}\right) / 2 \pi|x|^{2}$.

Proof. This is elementary and well known. If $f$ is radial, the derivative $(K * f \cdot \nabla)$ contains only the angular derivative. If $g$ is also radial, this implies $(K * f \cdot \nabla) g=0$. We thus conclude $b_{t}(f, g)=0$ so $B(f, g)=0$.

The second property of $B$ we need is the estimate for $B$. As we see later, it is convenient to divide the integral in $B$ into two parts - the region where $s$ is close to $t$ and $s$ is close to zero. We write

with

$$
\begin{aligned}
& B_{1}\left(w_{1}, w_{2}\right)(t)=\int_{0}^{t / 2} b_{t}\left(w_{1}, w_{2}\right)(\tau) d \tau \\
& B_{2}\left(w_{1}, w_{2}\right)(t)=\int_{t / 2}^{t} b_{t}\left(w_{1}, w_{2}\right)(\tau) d \tau
\end{aligned}
$$

so that

$$
b_{t}\left(w_{1}, w_{2}\right)(\tau)=-\nabla \cdot e^{(t-\tau) \Delta}\left(v_{1} w_{2}\right)(\tau), \quad v_{1}=K * w_{1}
$$

$$
B\left(w_{1}, w_{2}\right)=B_{1}\left(w_{1}, w_{2}\right)+B_{2}\left(w_{1}, w_{2}\right) .
$$

To simplify notation, for a function $f$ on $\mathbb{R}^{2} \times(0, T)$ we encode the decay in $t$ in norms:

$$
\begin{aligned}
& {[f]_{p T}=\sup _{0<t<T} t^{1-1 / p}\|f\|_{p},} \\
& {[f]_{p \delta T}=\sup _{0<t<T} t^{1-1 / p+\delta}\|f\|_{p} .}
\end{aligned}
$$

For example $[f]_{p T} \leqq 1$ for all $T$ is equivalent to $\|f\|_{p}(t) \leqq 1 / t^{1-1 / p}$ for all $t>0$. We estimate the decay rate of $B$ by using the decay rate of each variable in $B$.

Lemma 3.5. Suppose that $1 \leqq s \leqq p \leqq \infty, 1<q<2, \delta \geqq 0$ and $1 \leqq r \leqq \infty$ with $1 / s=1 / q+1 / r-1 / 2$. There is a positive constant $C$ depending only on $p, q, r$ and $\delta$ such that

$$
\left[B_{1}\left(w_{1}, w_{2}\right)\right]_{p \delta T} \leqq C \min \left(\left[w_{1}\right]_{q \delta T}\left[w_{2}\right]_{r T},\left[w_{1}\right]_{q T}\left[w_{2}\right]_{r \delta T}\right)
$$

with $\delta<1 / s-1 / 2$ and that

$$
\left[B_{2}\left(w_{1}, w_{2}\right)\right]_{p \delta T} \leqq C \min \left(\left[w_{1}\right]_{q \delta T}\left[w_{2}\right]_{r T},\left[w_{1}\right]_{q T}\left[w_{2}\right]_{r \delta T}\right),
$$

with $1 / s<1 / 2+1 /$ p. Here $w_{1}$ and $w_{2}$ are functions on $\mathbb{R}^{2} \times(0, T) ; B_{1}$ and $B_{2}$ are defined by (3.13).

Proof. Since $v_{1}=K * w_{1}$, we have by (3.3),

$$
\left\|v_{1}\right\|_{\theta} \leqq C_{1}\left\|w_{1}\right\|_{q} \quad 1 / \theta=1 / q-1 / 2, \quad 1<q<2 .
$$

Using Hölder's inequality, we have

$$
\left\|v_{1} w_{2}\right\|_{s} \leqq\left\|v_{1}\right\|_{\theta}\left\|w_{2}\right\|_{r} \leqq C_{1}\left\|w_{1}\right\|_{q}\left\|w_{2}\right\|_{r}, \quad 1 / s=1 / \theta+1 / r \leqq 1 .
$$

Applying (2.3b) now yields

$$
\begin{array}{rlrl}
\left\|b_{t}\left(w_{1}, w_{2}\right)\right\|_{p}(\tau) & \leqq C_{2}(t-\tau)^{-1 / 2-1 / s+1 / p}\left\|v_{1} w_{2}\right\|_{s}(\tau), & & p \geqq s \geqq 1 \\
& \leqq C_{3} M(t-\tau)^{-1 / 2-1 / s+1 / p} \tau^{-\sigma(q)-\sigma(r)-\delta}, & 0 \leqq \tau \leqq T,
\end{array}
$$


with $M=\left[w_{1}\right]_{q \delta T}\left[w_{2}\right]_{r T}$ or $\left[w_{1}\right]_{q T}\left[w_{2}\right]_{r \delta T}$, where $C_{j}$ is a constant depending only on $p, q$ and $r$ and $\sigma(q)=1-1 / q$. The restrictions on exponents $p, q, r$ we use so far are

$$
1 \leqq s \leqq p \leqq \infty, \quad 1<q<2, \quad 1 \leqq r \leqq \infty \quad \text { with } \quad 1 / s=1 / q+1 / r-1 / 2 .
$$

Since we see

$$
\begin{aligned}
& \int_{0}^{t / 2}(t-\tau)^{-\alpha} \tau^{-\beta} d \tau=c(\alpha, \beta) t^{-\alpha-\beta+1} \text { for } \beta<1 \\
& (c(\alpha, \beta): \text { constant independent of } t)
\end{aligned}
$$

by setting $\tau=t \tilde{\tau}$, the estimate (3.17) now yields

$$
\left\|B_{1}\left(w_{1}, w_{2}\right)\right\|_{p}(t) \leqq \int_{0}^{t}\left\|b_{t}\left(w_{1}, w_{2}\right)\right\|_{p}(\tau) d \tau \leqq C_{4} M t^{-\sigma(p)-\delta}, \quad t>0,
$$

provided that

$$
\sigma(q)+\sigma(r)+\delta<1,
$$

which is equivalent to

$$
\delta<1 / s-1 / 2 .
$$

The estimate (3.19) yields (3.15) under the restrictions (3.18) and (3.20).

It remains to prove (3.16). Since, as before,

$$
\int_{t / 2}^{t}(t-\tau)^{-\alpha} \tau^{-\beta} d \tau=c^{\prime}(\alpha, \beta) t^{-\alpha-\beta+1} \quad \text { for } \quad \alpha<1,
$$

the estimate (3.17) now yields

$$
\left\|B_{2}\left(w_{1}, w_{2}\right)\right\|_{p}(t) \leqq C_{5} M t^{-\sigma(p)-\delta}, \quad t>0,
$$

provided that

$$
1 / 2+1 / s-1 / p<1
$$

which is equivalent to

$$
1 / s<1 / 2+1 / p
$$

The estimate (3.21) yields (3.16) under (3.18) and (3.22) which completes the proof.

\section{Large Time Asymptotics of the Vorticity}

This section states our main results on the large time behavior of solutions of the vorticity equation.

Let $\omega_{0}$ be a finite Radon measure on $\mathbb{R}^{2}$, i.e. $\omega_{0} \in M\left(\mathbb{R}^{2}\right)$. We denote the total vorticity (at time zero) by

$$
\kappa=\int_{\mathbb{R}^{2}} \omega_{0}(d x) .
$$

We define the Reynolds number (at time zero) by

$$
R=v^{-1} \int_{\mathbb{R}^{2}}\left|\omega_{0}\right|(d x)=\left\|\omega_{0}\right\|_{1} / v,
$$


where $v>0$ is the kinematic viscosity which is assumed one in (3.1). The function

$$
\omega_{* \kappa}(x, t)=\frac{\kappa}{4 \pi v t} \exp \left(-|x|^{2} / 4 v t\right)=\kappa G(x, v t), \quad x \in \mathbb{R}^{2}
$$

is a solution of the heat equation

$$
\omega_{t}-v \Delta \omega=0
$$

with $\omega(x, 0)=\kappa \delta(x)$. Since $\omega_{* \kappa}$ is radially symmetric, Lemma 3.4 implies that $\left(v_{* \kappa} \cdot \nabla\right) \omega_{* \kappa}=0$, where $v_{* \kappa}=K * \omega_{* \kappa}$. We thus see $\omega_{* \kappa}$ solves the vorticity equation with $v>0$,

$$
\begin{gathered}
\omega_{t}-v \Delta \omega+(v \cdot \nabla) \omega=0, \\
v=K * \omega
\end{gathered}
$$

with initial data $\kappa \delta(x) \in M\left(\mathbb{R}^{2}\right)$. To avoid later confusion by a solution of the vorticity equation with $v$ we shall always mean a solution of (4.4)-(4.5) with (3.4) which satisfies (3.5)-(3.8) with $\omega(x, 0)=\omega_{0} \in M\left(\mathbb{R}^{2}\right)$. Since one can reduce (4.4)-(4.5) to (3.1)-(3.2) by a normalization, Proposition 3.1 guarantees the existence of such a solution for $\omega(x, 0)=\omega_{0} \in M\left(\mathbb{R}^{2}\right)$. For a solution $\omega$, (3.8) implies that the total vorticity is conserved for all time, namely

$$
\kappa=\int_{\mathbb{R}^{2}} \omega(x, t) d x, \quad t \geqq 0,
$$

where $\kappa$ is defined in (4.1). This says that the total vorticity is defined independent of time.

We claim that a solution $\omega$ of the vorticity equation with $v$ behaves like $\omega_{* \kappa}$ with the same total vorticity $\kappa$ provided the Reynolds number $R$ is small no matter what the initial vorticity $\omega_{0} \in M\left(\mathbb{R}^{2}\right)$ is.

Theorem 4.1. Suppose that the kinematic viscosity $v$ equals 1 and that $\omega(x, t)$ is a solution of the vorticity equation for $\omega(x, 0)=\omega_{0} \in M\left(\mathbb{R}^{2}\right)$. For every $\delta, 0<\delta<1 / 2$, there is $\varepsilon>0$ depending only on $\delta$ such that if the Reynolds number $R$ in (4.2) is smaller than $\varepsilon$, then

$$
\begin{gathered}
\left\|\omega-\omega_{* \kappa}\right\|_{p}(t) \leqq C N t^{-1+1 / p-\delta} \\
\left\|\omega-e^{t \Delta} \omega_{0}\right\|_{p}(t) \leqq C N t^{-1+1 / p-\delta}, \quad t>0, \quad 1 \leqq p \leqq \infty
\end{gathered}
$$

with a universal constant $C$ and $N=\left\|\left(|x|^{2}+1\right) \omega_{0}\right\|_{1}$, where $\kappa$ is the total vorticity of $\omega$ and $\omega_{* \kappa}$ is defined in (4.3).

We postpone to prove this theorem in the next section. Admitting Theorem 4.1, we derive various results. First, we observe that Theorem 4.1 gives the large time behavior of a solution $\omega(x, t)$ of the vorticity equation with $v>0$ just by a normalization. In fact $\bar{\omega}(x, t)=v^{-1} \omega(x, t / v)$ is a solution of the vorticity equation with $v=1$, where $\bar{\omega}(x, 0)=v^{-1} \omega(x, 0)$. Using this relation one can rewrite Theorem 4.1 for general $v>0$.

Theorem 4.2. Suppose that $\omega(x, t)$ is a solution of the vorticity equation with $v>0$ for $\omega(x, 0)=\omega_{0} \in M\left(\mathbb{R}^{2}\right)$. For every $\delta, 0<\delta<1 / 2$, there is $\varepsilon>0$ depending only on 
$\delta$ such that $R<\varepsilon$ implies

$$
\begin{gathered}
\left\|\omega-\omega_{* \kappa}\right\|_{p}(t) \leqq C N(t v)^{-1+1 / p-\delta} \\
\left\|\omega-e^{v t \Delta} \omega_{0}\right\|_{p}(t) \leqq C N(t v)^{-1+1 / p-\delta}, \quad t>0, \quad 1 \leqq p \leqq \infty
\end{gathered}
$$

with a universal constant $C$ and $N=\left\|\left(|x|^{2}+1\right) \omega_{0}\right\|_{1}$, where $\kappa$ is the total vorticity of $\omega$ and $\omega_{* \kappa}$ is defined in (4.3).

Since $\left\|\omega_{* \kappa}\right\|_{p}(t)=C_{p} \kappa(t v)^{-1+1 / p}$ by (2.1a), Theorems 4.1 and 4.2 give an asymptotic expression of a solution as $t \rightarrow \infty$. We at least observe that $\omega_{* \kappa}$ is the main term in the asymptotics as $t \rightarrow \infty$ and that the solution of the linear equation mainly describes the behavior as $t \rightarrow \infty$. We now discuss an asymptotic expansion of the velocity $v=K * \omega$ corresponding to (4.7a), (4.7b).

Theorem 4.3. Suppose that $\omega(x, t)$ is a solution of the vorticity equation with $v>0$ for $\omega(x, 0)=\omega_{0} \in M\left(\mathbb{R}^{2}\right)$. For every $\delta, 0<\delta<1 / 2$ there is $\varepsilon>0$ depending only on $\delta$ such that $R<\varepsilon$ implies

$$
\begin{aligned}
\left\|\nabla v-\nabla v_{* \kappa}\right\|_{q}(t) & \leqq C^{\prime} N(t v)^{-1+1 / q-\delta}, \quad N=\left\|\left(|x|^{2}+1\right) \omega_{0}\right\|_{1}, \\
\left\|\nabla v-\nabla e^{v t} v_{0}\right\|_{q}(t) & \leqq C^{\prime} N(t v)^{-1+1 / q-\delta}, \\
\left\|v-v_{* \kappa}\right\|_{r}(t) & \leqq C^{\prime \prime} N(t v)^{-1 / 2+1 / r-\delta}, \\
\left\|v-e^{v t} v_{0}\right\|_{r}(t) & \leqq C^{\prime \prime} N(t v)^{-1 / 2+1 / r-\delta} \quad t>0,
\end{aligned}
$$

with $C^{\prime}$ depending only on $q, 1<q<\infty$ and $C^{\prime \prime}$ depending only on $r, 2<r \leqq \infty$, where $v_{* \kappa}=K * \omega_{* \kappa}$ and $v_{0}=K * \omega_{0}$.

Proof. Since $\nabla K$ is the Calderon-Zygmund kernel, we have

$$
\|\nabla(K * f)\|_{q} \leqq C\|f\|_{q}, \quad 1<q<\infty,
$$

by applying the Calderon-Zygmund inequality [9, Chap. 9]. The estimate (4.7a, b) now yields $(4.8 \mathrm{a}, \mathrm{b})$. Estimate $(4.9 \mathrm{a}, \mathrm{b})$ for $2<r<\infty$ follows directly from $(4.7 \mathrm{a}, \mathrm{b})$ and (3.3).

It remains to prove $(4.9 \mathrm{a}, \mathrm{b})$ for $r=\infty$. Applying the Gagliardo-Nirenberg inequality (see e.g. [7, p. 24]) $\|g\|_{\infty} \leqq C\|g\|_{p}^{1-2 / p}\|\nabla g\|_{p}^{2 / p}$ for $p>2$ to $g=v-v_{* \kappa}$ or $v-e^{v t} v_{0}$, we see $(4.8 \mathrm{a}, \mathrm{b})$ and $(4.9 \mathrm{a}, \mathrm{b})$ for $2<r<\infty$ yield $(4.9 \mathrm{a}, \mathrm{b})$ for $r=\infty$.

Remark 4.4. There are several results $[2,10,14,18,20-22]$ on the decay of the velocity $v$ for the $n$-dimensional Navier-Stokes equations assuming that the initial velocity $v_{0}$ is in some $L^{p}$. When $n=2$, their main results read:

and

$$
\lim _{t \rightarrow \infty}\|v\|_{2}(t)=0 \quad \text { if } \quad v_{0} \in L^{2}\left(\mathbb{R}^{2}\right), \quad[10,14,18]
$$

$$
\begin{gathered}
\|v\|_{2}(t) \leqq C t^{-1 / q+1 / 2}, \quad\left\|v-e^{t \Delta} v_{0}\right\|_{2}(t) \leqq C t^{-1 / q+1 / 2-\delta} \\
v_{0} \in L^{2}\left(\mathbb{R}^{2}\right) \cap L^{q}\left(\mathbb{R}^{2}\right), \quad \delta=1 / q-1 / 2, \quad 1 \leqq q<2, \quad[10,20-22]
\end{gathered}
$$

where the viscosity $v$ is assumed one. The estimates (4.10) give an asymptotic expansion. Since our assumptions do not in general imply $v_{0} \in L^{2}\left(\mathbb{R}^{2}\right) \cap L^{q}\left(\mathbb{R}^{2}\right)$, 
(4.9b) is not included in (4.10). Also, (4.10) is not included in (4.9b) since $r=2$ is excluded in (4.9b). Among them Kato [14] studied the decay of $\|v\|_{r}(t), r>2$ other than energy. His results yield

$$
\begin{aligned}
& \|v\|_{r}(t) \leqq C t^{-1 / 2+1 / r} \quad 2 \leqq r \leqq \infty, \\
& \|\nabla v\|_{q}(t) \leqq C t^{-1+1 / q} \quad 2 \leqq q<\infty,
\end{aligned}
$$

provided $v_{0} \in L^{2}\left(\mathbb{R}^{2}\right)$. Our results $(4.8 \mathrm{a})$, (4.9a) claim the faster decay if $\kappa=\int \omega_{0}(d x)=0$.

\section{Proof of Theorem 4.1}

We study the integral equation (3.9):

$$
\omega(t)=e^{t \Delta} \omega_{0}+B(\omega, \omega) .
$$

A naive idea to prove (4.6b) would be to estimate $B(\omega, \omega)$. If we were successful to prove $\|B(\omega, \omega)\|_{p}(t) \leqq C t^{-1+1 / p-\delta}$, we would obtain (4.6b), and using the estimate for the linear part (2.6), (4.6b) would yield (4.6a). Unfortunately this idea appears not to work. In fact the optimal decay rate estimate for $\|\omega\|_{p}(t)$ is $t^{-1+1 / p}$ even if $\omega_{0}$ decays rapidly at infinity unless the total vorticity $\kappa=0$; the simplest example is $\omega_{* \kappa}$, where $\omega_{* \kappa}(x, 0)=\kappa \delta(x)$. Using Lemma 3.5, all we can derive from the estimate of $\|\omega\|_{p}$ is $\|B(\omega, \omega)\|_{p}(t) \leqq C t^{-1+1 / p}$ in general, which is too weak to derive (4.6b). To overcome this difficulty we would rather study the difference $w=\omega-\omega_{*}$, where $\omega_{*}=\omega_{* \kappa}$ in (4.3).

Proposition 5.1. Suppose that $\omega(x, t)$ is a solution of the vorticity equation for $\omega(x, 0)=\omega_{0} \in M\left(\mathbb{R}^{2}\right)$. Then the difference $w=\omega-\omega_{*}$ solves

$$
w=W+B(w, w)+B\left(w, \omega_{*}\right)+B\left(\omega_{*}, w\right),
$$

with

where

$$
W=e^{t \Delta} \omega_{0}-\omega_{*}, \quad \omega_{*}=\frac{\kappa}{4 \pi t} \exp \left(-\frac{|x|^{2}}{4 t}\right),
$$

$$
\kappa=\int_{\mathbb{R}^{2}} \omega_{0}(d x) .
$$

Proof. By Proposition 3.3 we may assume $\omega$ solves (3.9). Since $B$ is bilinear, plugging $\omega=w+\omega_{*}$ yields

$$
w=W+B(w, w)+B\left(w, \omega_{*}\right)+B\left(\omega_{*}, w\right)+B\left(\omega_{*}, \omega_{*}\right)
$$

Since $\omega_{*}$ is radial, Lemma 3.4 implies that the last term vanishes identically. We thus obtain (5.1).

We shall prove (4.6a) by estimating $W$ and $B$ in (5.1). Roughly speaking, we appeal to a perturbation method. We estimate $[w]_{p \delta T}$ by using the right-hand side of (5.1). Here $[w]_{p \delta T}$ is defined by (3.14) and is finite since $T<\infty$ and (3.5) holds; this is why we take $T<\infty$ rather than $T=\infty$. We eventually have

$$
[w]_{p \delta T} \leqq[W]_{p \delta T}+C R[w]_{p \delta T}, \quad(C \text { : constant })
$$


where $R$ is the Reynolds number. If $R$ is small enough, one gets

$$
[w]_{p \delta T} \leqq C^{\prime}[W]_{p \delta T}, \quad C^{\prime}>1 .
$$

Since $[W]_{p \delta T}$ is bounded independent of $T$ by (2.6), we conclude the desired estimate. Unfortunately, since there are restrictions of exponents in Lemma 3.5 such an idea only works for $p, 1<p<2$. After we prove (4.6a) for $1<p<2$, we shall prove it for $p=\infty$ and $p=1$ and interpolate these results. Even if we are interested only in the case $p=\infty$ or $p=1$, we should check the result for $1<p<2$.

Proposition 5.2. Suppose that $\omega(x, t)$ is a solution of the vorticity equation for $\omega(x, 0)=\omega_{0} \in M\left(\mathbb{R}^{2}\right)$ and that $1<p<2$. Then for $\delta, 0<\delta<1 / 2$ there is a constant $\varepsilon=\varepsilon(p, \delta)>0$ such that $R<\varepsilon$ implies

$$
[w]_{p \delta T} \leqq c N, \quad N=\left\|\left(|x|^{2}+1\right) \omega_{0}\right\|_{1}
$$

with a universal constant $c>0$, where $R$ is the Reynolds number and $w=\omega-\omega_{*}$, $\omega_{*}=\omega_{* \kappa}$. Here $\kappa$ is the total vorticity; $\kappa=\int \omega_{0}(d x)$.

Proof. We may assume $N<\infty$, otherwise the result is trivial. Since $1<p<2$, one can handle $B_{1}$ and $B_{2}$ simultaneously and estimates look symmetric for both variables in $B$. In fact, we take $s=1$ in (3.15) so that $\delta<1 / 2$. Since $1<p<2$ implies $1 / s=1<1 / 2+1 / p$, we can also apply (3.16). Taking $q=p$ or $r=p$ in (3.15) and (3.16) yields

$$
\left[B\left(w_{1}, w_{2}\right)\right]_{p \delta T} \leqq 2 C \min \left(\left[w_{1}\right]_{p \delta T}[w]_{\theta T},\left[w_{1}\right]_{\theta T}\left[w_{2}\right]_{p \delta T}\right)
$$

with $3 / 2=1 / \theta+1 / p$. Applying this to (5.1) we get

$$
[w]_{p \delta T} \leqq[W]_{p \delta T}+2 C[w]_{p \delta T}[w]_{\theta T}+4 C[w]_{p \delta T}\left[\omega_{*}\right]_{\theta T} .
$$

Since $T<\infty$, we note $[w]_{p \delta T}$ is finite. By (2.1a) we have

$$
\left[\omega_{*}\right]_{r T}=C_{r} \kappa \leqq c_{1} R \quad 1 \leqq r \leqq \infty,
$$

where $c_{j}(j=1,2 .$.$) is a universal constant. Since w=\omega-\omega_{*}$, for fixed $m>0$ the estimates (3.5) and (5.4) yield

$$
[w]_{r T} \leqq[w]_{r T}+\left[\omega_{*}\right]_{r T} \leqq\left(c_{1}+c_{2}\right) R \text { for } R \leqq m,
$$

where $c_{2}$ may depend on $m$. Applying (5.4) and (5.5) to (5.3) now yields

$$
[w]_{p \delta T} \leqq[W]_{p \delta T}+C^{\prime} R[w]_{p \delta T}
$$

with $C^{\prime}=2 C\left(3 c_{1}+c_{2}\right)$ depending only on $p$ and $\delta$. We take $\varepsilon$ sufficiently small, say,

$$
0<\varepsilon=\varepsilon(p, \delta)=\min \left(1 / 2 C^{\prime}, 1\right) .
$$

If $R<\varepsilon$, then (5.6) gives

$$
[w]_{p \delta T} \leqq 2[W]_{p \delta T} .
$$

The estimate (5.2) now follows from (2.6). 
If $p$ is large, one cannot take $s=1$ in (3.16), so we should split the integral in $B$ into two parts, $B_{1}$ and $B_{2}$. We next prove (5.2) for $p=\infty$.

Proposition 5.3. Suppose the same situation as in Proposition 5.2. For $\delta, 0<\delta<1 / 2$, there is a constant $\varepsilon_{\infty}>0$ such that $R<\varepsilon_{\infty}$ implies

$$
[w]_{\infty \delta T} \leqq c N,
$$

where $c$ is a universal constant.

Proof. We take $s=1$ and $r=q=4 / 3$ in (3.15), which gives

$$
\begin{aligned}
& {\left[B_{1}(w, w)\right]_{p \delta T} \leqq C[w]_{q \delta T}[w]_{q T}, \quad q=4 / 3,} \\
& {\left[B_{1}\left(w, \omega_{*}\right)\right]_{p \delta T}, \quad\left[B_{1}\left(\omega_{*}, w\right)\right]_{p \delta T} \leqq C[w]_{q \delta T}\left[\omega_{*}\right]_{q T},}
\end{aligned}
$$

where $\delta<1 / 2$. To estimate $B_{2}$, we take $s=4$ in (3.16) so that $1 / s<1 / 2 \leqq 1 / 2+1 / p$. Applying (3.16) with $q=4 / 3$ and $r=\infty$ yields

$$
\begin{aligned}
{\left[B_{2}(w, w)\right]_{\infty \delta T} } & \leqq C[w]_{q \delta T}[w]_{\infty T}, \\
{\left[B_{2}\left(w, \omega_{*}\right)\right]_{\infty \delta T} } & \leqq C[w]_{q \delta T}\left[\omega_{*}\right]_{\infty T}, \\
{\left[B_{2}\left(\omega_{*}, w\right)\right]_{\infty \delta T} } & \leqq C\left[\omega_{*}\right]_{q T}[w]_{\infty \delta T}, \quad q=4 / 3 .
\end{aligned}
$$

We apply (5.9) and (5.10) to (5.1) and use (5.4) and (5.5). Similarly to (5.6), we obtain

$$
[w]_{\infty \delta T} \leqq[W]_{\infty \delta T}+C_{1} R[w]_{q \delta T}+C_{2} R[w]_{\infty \delta T},
$$

where $q=4 / 3, C_{1}=2 C\left(3 c_{1}+c_{2}\right), C_{2}=c_{1} C$. We take

$$
\varepsilon_{\infty}=\min \left(\varepsilon(4 / 3, \delta), 1 / 2 C_{2}, 1 / 2 C_{1}\right) \text {. }
$$

If $R<\varepsilon_{\infty}$, the estimate (5.11) together with (5.2) now yields

$$
[w]_{\infty \delta T} \leqq 2[W]_{\infty \delta T}+c N .
$$

Applying (2.6), we now obtain

$$
[w]_{\infty \delta T} \leqq 2 c N,
$$

which is the same as (5.8) by replacing $2 c$ by $c$.

Unfortunately we are forced to treat the case $p=1$ separately because one cannot take $s=4$ in (3.16) which requires $p \geqq s=4$.

Proposition 5.4. Suppose the same situation as in Proposition 5.2. For $\delta, 0<\delta<1 / 2$, there is a constant $\varepsilon_{1}>0$ such that $R<\varepsilon_{1}$ implies

$$
[w]_{1 \delta T} \leqq c N,
$$

where $c$ is a universal constant.

Proof. We observe that (5.9) holds even for $p=1$. However, we need modification to (5.10). We take $s=1$ and $r=q=4 / 3$ in (3.16) which yields the estimate (5.9) where $B_{1}$ is replaced by $B_{2}$. Similarly to deriving (5.6), one gets

$$
[w]_{1 \delta T} \leqq[w]_{1 \delta T}+2 C_{1}^{\prime} R[w]_{q \delta T}, \quad q=4 / 3,
$$


with $C_{1}^{\prime}=2 C\left(3 c_{1}+c_{2}\right)$. We take

$$
\varepsilon_{1}=\min \left(\varepsilon(4 / 3, \delta), 1 / 2 C_{1}^{\prime}\right) .
$$

Applying (5.2) with (2.6), we observe

$$
[w]_{1 \delta T} \leqq[W]_{1 \delta T}+c N \leqq 2 c N,
$$

provided $R<\varepsilon_{1}$. This is the same as $(5.12)$ by replacing $2 c$ by $c$.

We now obtain similar estimates like (5.2) for all $1 \leqq p \leqq \infty$, just by an interpolation.

Proposition 5.5. Suppose the same situation as in Proposition 5.2. For $\delta, 0<\delta<1 / 2$, there is $\varepsilon$ depending only on $\delta$ such that $R<\varepsilon$ implies

$$
[w]_{r \delta T} \leqq c N, \quad 1 \leqq r \leqq \infty,
$$

where $c$ is a universal constant.

Proof. The Riesz-Thorin interpolation (see e.g. [4]) yields

$$
[w]_{r \delta T} \leqq[w]_{1 \delta T}^{1 / r}[w]_{\infty \delta T}^{1-1 / r}, \quad 1 \leqq r \leqq \infty .
$$

Interpolating (5.8) and (5.12) now shows that $[w]_{r \delta T} \leqq c N$, provided $R<\varepsilon=$ $\min \left(\varepsilon_{1}, \varepsilon_{\infty}\right)$.

Proof of Theorem 4.1. Since $c$ is independent of $T$, (5.13) yields (4.6a) by the definition of the norm (3.14).

It remains to prove $(4.6 \mathrm{~b})$. Since

$$
\omega-e^{t \Delta} \omega_{0}=\omega-\omega_{*}-W=w-W,
$$

(4.6b) follows immediately from (4.6a) and (2.6).

\section{Stability of Burgers' Vortex-Formation of a Concentrated Vortex}

This section is devoted to an application of our large time asymptotic expression for the vorticity (Theorem 4.2). We consider a three-dimensional viscous incompressible flow written as a superposition of an axisymmetric irrotational flow and a two-dimensional flow whose vorticity vector directs to the symmetry axis. We study the large time behavior of such a flow when the axisymmetric flow is a inward convection-axially constant stretching flow. We shall show the vorticity tends to Burgers' vortex $[1,5]$ as the time tends to infinity provided the Reynolds number of two-dimensional flow is small. No particular structure of initial vorticity is assumed. There are no assumptions on the speed of the axisymmetric flow. Our asymptotic results physically imply formation of a concentrated vortex.

We consider the Navier-Stokes equations in the three dimensional space $\mathbb{R}^{3}$ :

$$
\frac{\partial u}{\partial \tau}-v \Delta u+(u \cdot \nabla) u+\nabla p=0, \quad \nabla \cdot u=0
$$

where $u=u(y, \tau), p=p(y, \tau), y=\left(y_{1}, y_{2}, y_{3}\right)$. Suppose that our velocity field $u$ is 
expressed as

$$
u=U+V \text {, }
$$

$U$ : an axisymmetric irrotational divergence-free velocity field,

$V$ : a two-dimensional rotational velocity field,

where the vorticity of $u$ (or $V$ ) directs to the symmetry axis. To fix the idea, we take $y_{3}$-axis as the symmetry axis. The vector field $U$ is assumed to have the form

$$
U\left(y_{1}, y_{2}, y_{3}, \tau\right)=\left(-\alpha y_{1},-\alpha y_{2}, 2 \alpha y_{3}\right) \alpha=\alpha(\tau)
$$

which evidently satisfies $\nabla \cdot U=0$ and $\nabla \times U=0$. If $\alpha$ is a positive constant, $U$ is a steady convection-axially stretching flow. The vector field $V$ has the form

$$
V\left(y_{1}, y_{2}, y_{3}, \tau\right)=\left(V^{1}\left(y_{1}, y_{2}, \tau\right), V^{2}\left(y_{1}, y_{2}, \tau\right), 0\right)
$$

so that the vorticity is

$$
\left(0,0, \Omega\left(y_{1}, y_{2}, \tau\right)\right)
$$

where $\Omega=\nabla \times V=\left(\partial / \partial y_{1}\right) V^{2}-\left(\partial / \partial y_{2}\right) V^{1}$. We first derive the vorticity equation for $\Omega$.

Proposition 6.1. The equations (6.1) with (6.2)-(6.5) are formally equivalent to

$$
\begin{gathered}
\frac{\partial \Omega}{\partial \tau}-v \Delta \Omega-\alpha(y \cdot \nabla) \Omega-2 \alpha \Omega+(V \cdot \nabla) \Omega=0, \\
V=K * \Omega, \quad K=\left(-y_{2}, y_{1}\right) / 2 \pi|y|^{2},
\end{gathered}
$$

provided that $\alpha$ is a constant and that $V$ decays at space infinity.

Proof. This is very similar to the proof of the equivalence of the vorticity equation and the Navier-Stokes equation. Plugging $u=U+V$ in (6.1), and noting

we obtain

$$
\begin{gathered}
(U \cdot \nabla) U=\nabla P, \quad P=\alpha^{2}\left(y_{1}^{2}+y_{2}^{2}+4 y_{3}^{2}\right) / 2, \\
(V \cdot \nabla) U=-\alpha V, \quad(U \cdot \nabla) V=-\alpha(y \cdot \nabla) V,
\end{gathered}
$$

$$
\frac{\partial V}{\partial \tau}-\nu \Delta V-\alpha(y \cdot \nabla) V-\alpha V+(V \cdot \nabla) V+\nabla(P+p)=0, \quad \nabla \cdot V=0 .
$$

Taking $\nabla \times$ of (6.8) yields (6.6), since

$$
\nabla \times((y \cdot \nabla) V)=(y \cdot \nabla) \Omega+\Omega .
$$

Since $\nabla \cdot V=0$ and $V$ decays at $|y|=\infty$, we have (6.7). This shows that (6.1) with (6.2)-(6.5) yields (6.6) and (6.7). Since the above calculation shows that (6.6) implies that $\partial u / \partial \tau-v \Delta u+(u \cdot \nabla) u$ is irrotational, (6.1) now follows from (6.7).

Our main goal is to study the large time asymptotic behavior of the vorticity $\Omega$ of (6.6)-(6.7) with arbitrary initial data $\Omega(y, 0)=\Omega_{0} \in M\left(\mathbb{R}^{2}\right)$. If $\alpha=0$, Theorem 4.2 already gives an answer, since (6.6)-(6.7) is nothing but (4.4)-(4.5). The vorticity $\Omega(y, \tau)$ is asymptotically equivalent to $\omega_{* \kappa}$ in $(4.3)$ called the diffused vortex filament of Oseen [17] with the total vorticity $\kappa$ provided that the Reynolds number $R$ is 
sufficiently small. We shall always assume that $\alpha$ is a positive constant unless otherwise claimed. As before, we define the Reynolds number $R$ and the total vorticity $\kappa$ by

$$
R=v^{-1} \int\left|\Omega_{0}\right|(d y)=v^{-1}\left\|\Omega_{0}\right\|_{1}, \quad \kappa=\int \Omega_{0}(d y) .
$$

We observe that (6.6)-(6.7) has a special steady (circular symmetric) solution called Burgers' vortex $[1,5]$ :

$$
\bar{\Omega}_{\kappa}=\frac{\kappa}{\pi l^{2}} e^{-|y|^{2} / l^{2}}, \quad l=(2 v / \alpha)^{1 / 2} .
$$

We shall claim below that $\Omega$ in (6.6)-(6.7) converges to $\bar{\Omega}_{\kappa}$ as $\tau \rightarrow \infty$ provided the Reynolds number $R$ is small.

Theorem 6.2. For a general initial vorticity $\Omega_{0} \in M\left(\mathbb{R}^{2}\right)$, there is a solution $\Omega(y, \tau)$ of (6.6)-(6.7) having the following properties. For every $\delta, 0<\delta<1 / 2$, there is $\varepsilon_{\delta}>0$ (independent of $\alpha, p$ and $\tau)$ such that

$$
\left\|\Omega(y, \tau)-\bar{\Omega}_{\kappa}(y)\right\|_{p}=0\left(e^{-2 \alpha \delta \tau}\right) \quad \text { as } \tau \rightarrow \infty,
$$

where $1 \leqq p \leqq \infty$, provided $R<\varepsilon_{\delta}$. Here $\bar{\Omega}_{\kappa}$ is Burgers' vortex defined in (6.10) and $\kappa$ is the total vorticity defined in (6.9). Moreover, the total vorticity is conserved, i.e.,

$$
\kappa=\int \Omega(y, \tau) d y \text { for all } \tau \geqq 0 .
$$

Proof. We first observe that (6.6)-(6.7) can be reduced to (4.4)-(4.5) by a time-dependent scaling transformation which is introduced by Lundgren [16] and by Kambe $[11,13]$ for the axially symmetric case. We introduce $x, t, \omega(x, t)$ such that

$$
\begin{aligned}
x & =A(\tau) y, \quad t=\int_{0}^{\tau} A^{2}(\sigma) d \sigma, \quad \omega(x, t)=A^{-2}(\tau) \Omega(y, \tau), \\
A^{\prime} & =\frac{d}{d \tau} A(\tau)=\alpha A, \quad A(0)=1 .
\end{aligned}
$$

Since $d t=A^{2}(\tau) d \tau, d x=A(\tau) d y$, we have

$$
\begin{aligned}
\partial_{t} \omega(x, t) & =A^{-2} \partial_{\tau}\left(\Omega(x / A(\tau), \tau) \cdot A^{-2}\right)=A^{-4}\left(\partial_{\tau} \Omega-A^{\prime} A^{-1}(y, \nabla) \Omega-2 A^{\prime} A^{-1} \Omega\right), \\
\Delta \omega(x, t) & =A^{-4} \Delta_{y} \Omega, \\
(v \cdot \nabla) \omega & =A^{-4}\left(V \cdot \nabla_{y}\right) \Omega \quad \text { with } \quad v=V / A(\tau) .
\end{aligned}
$$

Observing $A^{\prime}=\alpha A$, we see $\omega$ solves (4.4). By a dilation of the variable of the integration, we obtain (4.5) by putting $v=V / A(\tau)$. (The transformation (6.13) reduces (6.6)-(6.7) to (4.4)-(4.5) even if $\alpha$ is time dependent.)

Suppose that $\omega(x, t)$ is a solution of the vorticity equation with $v$ whose existence is proved in Proposition 3.1 with an appropriate scaling. Since $A(0)=1$ implies $\omega_{0}=\omega_{0}(x, 0)=\Omega_{0}(x, 0)=\Omega_{0}(y, 0)$, our asymptotic result (4.7a) yields

$$
\left\|\omega-\omega_{* \kappa}\right\|_{p}(t) \leqq C N(t v)^{-1+1 / p-\delta}, \quad 0<\delta<1 / 2, \quad 1 \leqq p \leqq \infty,
$$

provided that $R=v^{-1}\left\|\omega_{0}\right\|_{1}=v^{-1}\left\|\Omega_{0}\right\|_{1}$ is sufficiently small, say, $R<\varepsilon_{\delta}$, where $\varepsilon=\varepsilon_{\delta}$ is the same as in Theorem 4.2. Using original variables $y, \tau, \Omega$ in (6.13), this 
estimate yields

$$
\left\|\Omega-\Omega_{* \kappa}\right\|_{p}(\tau)\left(=\left(\int_{\mathbb{R}^{2}}\left|\Omega(y, \tau)-\Omega_{* \kappa}(y, \tau)\right|^{p} d y\right)^{1 / p}\right) \leqq C N\left(A(\tau)^{2} / v t\right)^{1-1 / p}(v t)^{-\delta}
$$

where

$$
\Omega_{* \kappa}(y, \tau)=\frac{\kappa}{\pi l^{2}\left(1-e^{-2 \alpha \tau}\right)} \exp \left(-\frac{|y|^{2}}{l^{2}\left(1-e^{-2 \alpha \tau}\right)}\right), \quad l=(2 v / \alpha)^{1 / 2},
$$

since $t=\left(e^{2 \alpha \tau}-1\right) / 2 \alpha, A=e^{\alpha \tau}$. Since

we have

$$
\lim _{\tau \rightarrow \infty} A(\tau)^{2} / v t=4 / l^{2}
$$

$$
\limsup _{\tau \rightarrow \infty} e^{2 \alpha \delta \tau}\left\|\Omega-\Omega_{* \kappa}\right\|_{p}(\tau) \leqq \gamma C N, \quad \gamma=\left(4 l^{-2}\right)^{1-1 / p+\delta} .
$$

In particular

$$
\left\|\Omega-\Omega_{* \kappa}\right\|_{p}(\tau)=0\left(e^{-2 \alpha \delta \tau}\right), \quad 0<\delta<1 / 2
$$

as $\tau \rightarrow \infty$. Clearly,

$$
\lim _{\tau \rightarrow \infty} \Omega_{* \kappa}(y, t)=\bar{\Omega}_{\kappa}(y),
$$

where $\bar{\Omega}_{\kappa}$ is Burgers' vortex in (6.10). More precisely, a direct calculation to (6.14) shows that

$$
\left\|\Omega_{* \kappa}-\bar{\Omega}_{\kappa}\right\|_{p}=0\left(e^{-2 \alpha \tau}\right) \quad \text { as } \tau \rightarrow \infty .
$$

The estimates (6.15) and (6.16) now yield (6.11).

It remains to prove $(6.12)$. Since

$$
\int \omega(x, t) d x=\int \Omega(y, \tau) d y,
$$

(3.8) now yields (6.12).

Remark. For radial initial data $\Omega_{0}$ the estimate (6.11) is pointed out by Kambe [12] at least for $p=\infty$ without the assumption on the Reynolds number. In this case by Lemma 3.4, $(V \cdot \nabla) \Omega$ in (6.6) vanishes so the problem is reduced to the heat equation. For the heat equation (2.6) shows that (4.7a) holds even for large $R$. Parallel to the above proof, we see (6.11) holds without the assumption on $R$ which extends results in [12].

\section{References}

1. Batchelor, G. K.: An introduction to fluid dynamics. Cambridge: Cambridge University Press $1967, \S 5.2$

2. Beirão da Veiga, H.: Existence and asymptotic behavior for strong solutions of the Navier-Stokes equations in the whole space. Indiana Univ. Math. J. 36, 149-166 (1987)

3. Benfatto, G., Esposito, R., Pulvirenti, M.: Planar Navier-Stokes flow for singular initial data. Nonlinear Anal. 9, 533-545 (1985)

4. Bergh, J., Löfström, J.: Interpolation spaces. An introduction. Berlin, Heidelberg, New York: Springer 1976 
5. Burgers, J. M.: A mathematical model illustrating the theory of turbulence. Adv. Appl. Mech. 1, 171-199 (1948)

6. Friedman, A.: Partial differential equations of parabolic type. New Jersey: Prentice Hall 1964

7. Friedman, A.: Partial differential equations. New York: Holt Rinehart and Winston 1969

8. Giga, Y., Miyakawa, T., Osada, H.: Two dimensional Navier-Stokes flow with measures as initial vorticity. Arch. Ration. Mech. Anal. (to appear)

9. Gilbarg, D., Trudinger, N. S.: Elliptic partial differential equations of second order, 2nd ed. Berlin, Heidelberg, New York: Springer 1983

10. Kajikiya, R., Miyakawa, T.: On $L^{2}$ decay of weak solutions of the Navier-Stokes equations in $\mathbb{R}^{n}$. Math. Z. 192, 135-148 (1986)

11. Kambe, T.: A class of exact solutions of two-dimensional viscous flow. J. Phys. Soc. Jpn. 52, 834-841 (1983)

12. Kambe, T.: Axisymmetric vortex solution of Navier-Stokes equation. J. Phys. Soc. Jpn. 53, 13-15 (1984)

13. Kambe, T.: A class of exact solutions of the Navier-Stokes equation. Fluid Dyn. Res. 1, 21-31 (1986)

14. Kato, T.: Strong $L^{p}$-solutions of the Navier-Stokes equation in $\mathbb{R}^{n}$ with applications to weak solutions. Math. Z. 187, 471-480 (1984)

15. Ladyzhenskaya, O. A., Solonnikov, V. A., Ural'ceva, N. N.: Linear and Quasilinear Equations of Parabolic Type. Trans. Math. Monogr., vol. 23, Providence, R. I.: Am. Math. Soc. 1968

16. Lundgren, T. S.: Strained spiral vortex model for turbulent fine structure. Phys. Fluids 25, 2193-2203 (1982)

17. Oseen, C. W.: Über Wirbelbewegung in einer reibenden Flüssigkeit. Ark. Mat. Astr. Fys. 7, $1-13$ (1911)

18. Masuda, K.: Weak solutions of the Navier-Stokes equations. Tohoku Math. J. 36, 623-646 (1984)

19. Reed, M., Simon, B.: Methods of Modern Mathematical Physics, Vol. II. New York: Academic Press 1975

20. Schonbek, M. E.: $L^{2}$ decay for weak solutions of the Navier-Stokes equations. Arch. Ration. Mech. Anal. 88, 209-222 (1985)

21. Schonbek, M. E.: Large time behaviour of solutions to the Navier-Stokes equations. Comm. PDE 11, 733-763 (1986)

22. Wiegner, M.: Decay results for weak solutions of the Navier-Stokes equations on $\mathbb{R}^{n}$. J. Lond. Math. Soc. 35, 303-313 (1987)

Communicated by J. L. Lebowitz

Received June 1, 1987

Note added in the proof. Professor Kawashima kindly pointed out that the right-hand side of (2.6) can be replaced by $C t^{-\sigma(p)-1 / 2}\||x| a\|_{1}$. A proof for $n=1$ is found in Lemma 8.1 of his article published in Proc. Royal Soc. of Edinburgh, 106A, 169-194, 1987 and it works for several space dimensions with necessary modifications. By this remark one can replace $\left(|x|^{2}+1\right) \omega_{0}$ by $|x| \omega_{0}$ in the definition of $N$ in Sect. 4, 5 and 6. 